\title{
Assembly of nuclear pore complexes in Xenopus egg extract
}

\author{
Marie-Christine Dabauvalle, Ulrich Scheer \\ Institute of Zoology I, University of Würzburg, Röntgenring 10, D-8700 Würzburg, Germany \\ (Received 18 February 1991; accepted 15 March 1991)
}

The nuclear pore complex : structural, functional and dynamic aspects

In eukaryotic cells, chromatin is enclosed by a doublelayered membrane system, the nuclear envelope, which segregates the nuclear genome and its transcriptional apparatus from the cytoplasm. Major structural elements of the nuclear envelope include inner and outer nuclear membrane, a lamina composed of a network of intermediate filament-related proteins underlying the inner nuclear membrane and numerous pore complexes traversing the perinuclear cisterna (for recent reviews see $[15,26])$. Pore complexes provide plasmatic channels through the nuclear membrane barrier for nucleocytoplasmic exchange of proteins and RNA/RNP [13]. It is now well established that proteins destined for the nucleus (karyophilic proteins) must possess a specific localization signal sequence for their selective transport through the pore complexes (for recent review see [29]). By high resolution mapping of such karyophilic proteins en route through the pore center, localization of antibodies against specific pore complex constituents using immunogold electron microscopy, application of image processing techniques and quantitative electron microscopy, considerable progress has been made in the understanding of the functional organization of pore complexes at the molecular level (eg $[1,25])$.

A family of pore complex glycoproteins with cytoplasmically or nucleoplasmically exposed O-linked $\mathrm{N}$ acetylglucosamine (GlcNAc) residues, whose members are located within the pore channel proper, appear to be part of the pore transport machinery. Thus, wheat germ agglutinin (WGA; a lectin which binds to terminal GlcNAcmoieties) as well as monoclonal antibodies against GlcNAc-modified pore proteins ("nucleoporins") have been shown to inhibit nuclear uptake of karyophilic proteins both in vivo and in vitro (for review see [29]).

Due to the selective import of karyophilic proteins and exclusion of karyophobic proteins, the nuclear compartment has a protein composition that is markedly different from that of the cytoplasm (for details see [8,9]). However, during the "open" mitosis of higher eukaryotic cells this nucleocytoplasmic compartmentalization breaks down concomitantly with the disassembly of the nuclear envelope. The specific molecular environment within the postmitotic daughter nuclei is then progressively restored after reformation of the nuclear envelope by selective uptake of those nuclear proteins that became distributed throughout the cytoplasm during mitosis. The newly formed pore complexes play an essential part in this remigration of nuclear proteins. When pore-mediated transport is inhibited by microinjection of WGA or a porespecific monoclonal antibody (directed aginst p68; see below), daughter nuclei become enclosed by a nuclear envelope with pore complexes but yet remain arrested in a telophase-like situation, ie are unable to enlarge, to decondense their chromosomes and to reform nucleoli $[5,6]$.

The rapid and efficient assembly of functional pore complexes is thus of fundamental importance for postmitotic cells to re-establish interphase nuclear organization and to enter the G1-phase. Nuclear envelope reconstitution involves the coordinate interactions of nuclear membrane vesicles, depolymerized lamina constituents and disassembled pore complex material, all of which are distributed throughout the cytoplasm of mitotic cell.

\section{In vitro assembly of the nuclear envelope}

Analyses of the mode and order of interaction of the disassembled nuclear envelope components with each other and the chromosome surface was greatly facilitated by the introduction of cell-free systems capable to assembly nuclei around added DNA or chromatin [7, 20-23]. Extracts from Xenopus eggs contain large amounts of the disassembled component parts required for de novo formation of nuclear envelopes such as depolymerized lamin $\mathrm{L}_{3}$ (the only lamin protein present in these cells $[4,31])$, membrane vesicles competent for nuclear membrane formation [32] and soluble pore complex proteins in form of specific macromolecular assemblies [11]. Such in vitro systems may be further subfractionated, depleted of specific components by immunoadsorption or otherwise manipulated in order to purify the essential components involved in the assembly process and to study their molecular interactions.

\section{The soluble form of the pore-specific protein p68}

We have studied the soluble form of the pore-specific protein p68 in Xenopus eggs. In the assembled pore complex this major GlcNAc-modified protein is located in the pore channel and involved in nuclear transport of proteins [10]. Most likely p68 is the Xenopus counterpart of the rat liver pore complex glycoprotein p62, whose primary sequence has been recently determined [30]. After centrifugation, p68 is recovered in the high speed supernatant of Xenopus egg extract indicating that this pore complex protein is not associated with membrane vesicles. However, p68 
does not occur in a monomeric form but rather as a larger and stable macromolecular complex containing, besides p68, a defined set of other proteins [11]. This finding agrees with the distinctly punctate immunofluorescent pattern in the cytoplasm of mitotic cells seen with a monoclonal antibody against p68 [5]. Obviously, nuclear pore complexes do not completely disintegrate during mitosis but remain at least partly assembled.

When Xenopus egg extract is immunodepleted of the p68-containing complex, chromatin aquires a continuous double-membrane envelope which, however, lacks pore complexes. Furthermore, these pore-deficient nuclei are unable to take up proteins from the surrounding medium [11]. Other authors who have used extracts depleted of WGA-binding proteins also found that the in vitro reconstituted nuclei acquired a nuclear envelope and were defective for transport [14]. In contrast to our results, however, Finlay and Forbes did observe pores in the nuclear envelope of the depleted nuclei and therefore concluded that the "nucleoporin-family" is required for active transport processes but not for assembly of the pore complex structure [14]. We think that differences of the templates used for nuclear assembly caused the divergent results. While we used protein-free bacteriophage lambda DNA which, upon incubation in the egg extract is first assembled into nucleosomes and then into chromatin before it can serve as a template for nuclear envelope assembly [23], Finlay and Forbes [14] added demembranated sperm nuclei. Since it is well known that solubilization of nuclear membranes leaves behind a lamina-pore complex supramolecular structure (for examples see [26]), it is unclear whether the pore complexes described in this work are in fact newly assembled structures.

Our results demonstrate that assembly of a continuous double-membrane nuclear envelope does not require concomitant pore complex formation. Hence they do not support the recently proposed "prepore model" which postulates that nascent pore complexes first have to bind to the chromatin surface in order to provide anchoring sites for nuclear membrane vesicles [28].

\section{Assembly of pore complexes in the absence of chromatin}

It is generally assumed that assembly of a nuclear envelope requires the concerted interaction of chromatin, membrane vesicles and soluble lamina and pore complex components (see [19] for review). We have examined the role of chromatin in this process by incubating Xenopus egg extract in the absence of DNA or chromatin. Quite surprisingly under these conditions a large number of nuclear envelope-like membranes with densely spaced pore complexes were formed (fig 1; for details see [12]). These "'annulate lamellae" occur in form of individual flattened membrane cisternae or large stacks of parallel sheets, often in concentric circular arrangements (fig 1). Apart from their tendency to form multilamellar stacks, their somewhat higher pore density and the absence of chromatin, annulate lamellae are indistinguishable from nuclear envelopes. This holds true in particular for the pore complexes based on ultrastructural and immunolocalization data [12]. In essence, our results indicate that nuclear membrane vesicles have the ability to fuse spontaneously into flattened membrane cisternae and to acquire pore complexes independently of any interactions whith chromatin and a lamina. How the soluble non-membranous pore material interacts with the membranes to form a pore complex is at present unknown. However, it is tempting to speculate that the transmembrane glycoprotein gp210 which is likely to be located at the periphery of pore complexes [17, 34] might be involved.

When we added increasing amounts of chromatin to the extract, nuclear envelopes formed at the expense of annulate lamellae until, at sufficiently high chromatin concentrations, annulate lamellae were no longer detectable [12]. Together, these observations show that the extract will assemble spontaneously pore complex-containing membranes. Whether and in what proportion they occur in form of annulate lamellae or nuclear envelopes is determined by the chromatin surface available for interaction with the nascent membranes.

\section{On the role of the lamina}

Is the lamina involved in targeting nuclear membrane vesicles to the chromatin surface? In order to address this question we have added antibodies to lamin $\mathrm{L}_{3}$ to Xenopus egg extract at concentrations that efficiently inhibited lamina assembly. Whereas annulate lamellae formed normally under these conditions, reconstitution of nuclear envelopes around added DNA was completely inhibited [12]. Thus, a lamina is clearly required for targeting the nascent nuclear membrane vesicles to the chromosome surface whereas membrane fusion and assembly of pore complexes are lamina-independent processes.

Does the lamina retain its potential to spatially direct nuclear envelope assembly in the absence of chromatin? Normally the assembly of a supramolecular lamina structure is promoted by specific lamin-chromatin interactions and hence restricted to chromosome surfaces [16]. However, at sufficiently high concentrations lamins will also polymerize in vitro in the absence of chromatin into filamentous structures [16]. We have assembled in vitro Xenopus lamin A [33] into filaments and incubated the resulting aggregates in egg extract without addition of DNA or chromatin (lamin A was expressed in $E$ coli, purified, solubilized in urea and kindly provided by Georg Krohne). After a $60 \mathrm{~min}$ incubation, the chromatin-free lamin aggregates were partially coated with nuclear envelope fragments containing pore complexes (fig 2). Lamin A filaments thus have the intrinsic property to bind nascent nuclear envelopes. Integral membrane proteins specific to the inner nuclear membrane might be involved in such lamina-membrane interactions [27].

In an earlier study Burke and Gerace [7] reported that immunodepletion of lamins from mitotic mammalian cell extract inhibited subsequent membrane and pore complex formation around chromosomes. Our results confirm and extend this notion since we can now define more precisely the role of the lamina in this assembly process. In contrast, in a recent study Newport et al [24] proposed a laminindependent pathway for nuclear envelope assembly in Xenopus egg extract. However, in this context it has to be kept in mind that demembranated Xenopus sperm nuclei used as a chromatin source in this study contain an endogenous lamina (formed by lamin $\mathrm{L}_{4}$ [2]) which might be sufficient to bind nascent nuclear membrane vesicles.

The assembly pathway of the nuclear envelope and nuclear envelope-like structures (annulate lamellae) in Xenopus egg extract is schematically depicted in figure 3. According to this model, fusion of nuclear membrane vesicles and assembly of pore complexes occur spontaneously, independent of any interactions with chromatin or 


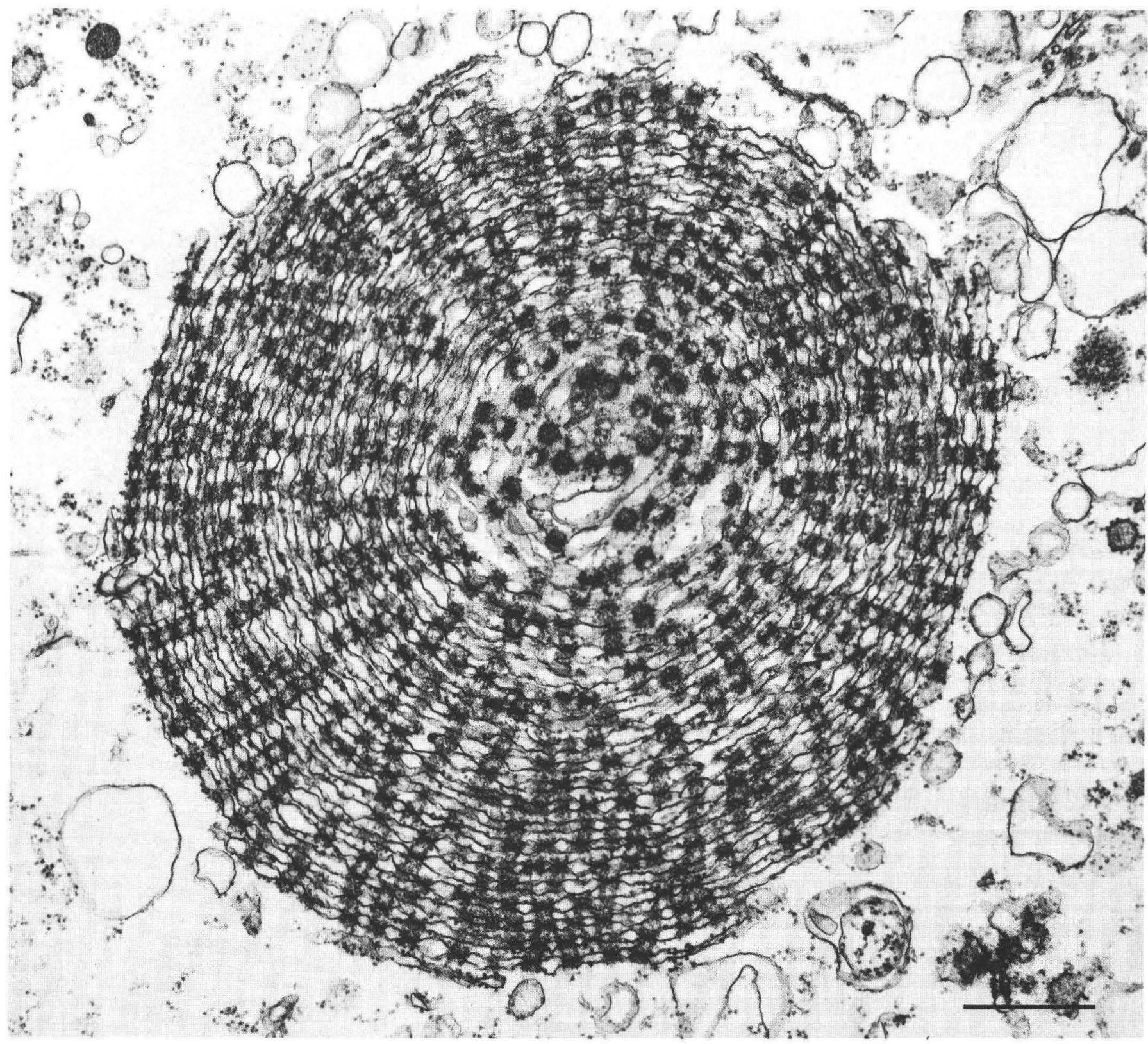

Fig 1. Electron micrograph showing a stack of spontaneously assembled annulate lamellae in Xenopus egg extract after 90 min incubation in the absence of DNA. Although this is an exceptionally striking example, such circular concentric arrangements of annulate lamellae are frequently seen. Note that pores in adjacent lamellae are often aligned. Bar $=0.5 \mu \mathrm{m}$.

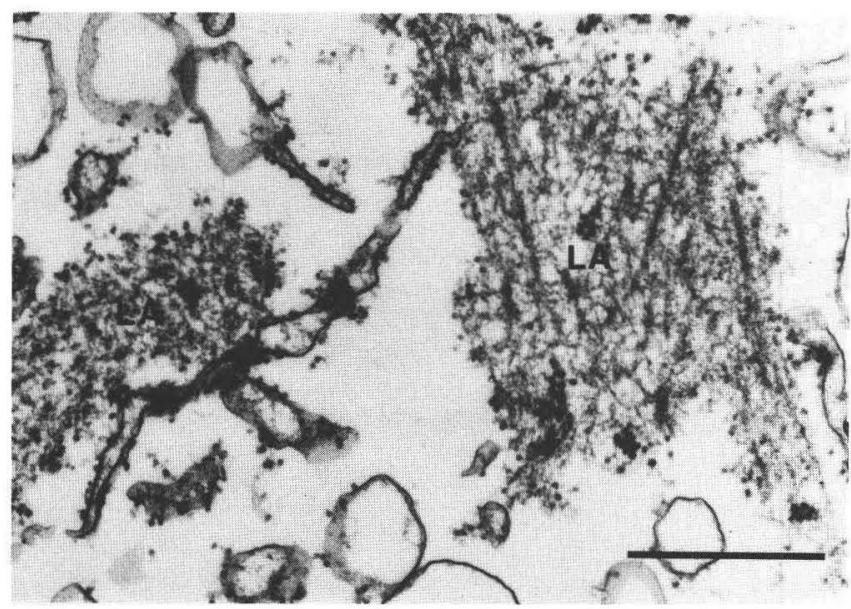

Fig 2. Incubation of in vitro assembled Xenopus lamin Afilaments in egg extract in the absence of DNA. Purified lamin A [33] (the lamin protein was expressed in E coli, purified, solubilized in urea and kindly provided by Georg Krohne) was dialyzed against $83 \mathrm{mM} \mathrm{KCl}, 17 \mathrm{mM} \mathrm{NaCl}, 10 \mathrm{mM}$ phosphate buffer (pH 7.4) to allow spontaneous assembly into filamentous lamin aggregates. They were then added to egg extract and analysed $60 \mathrm{~min}$ later by electron microscopy. Numerous lamin aggregates (LA) are associated with flattened membrane cisternae containing pore complexes. Bar $=0.5 \mu \mathrm{m}$. 


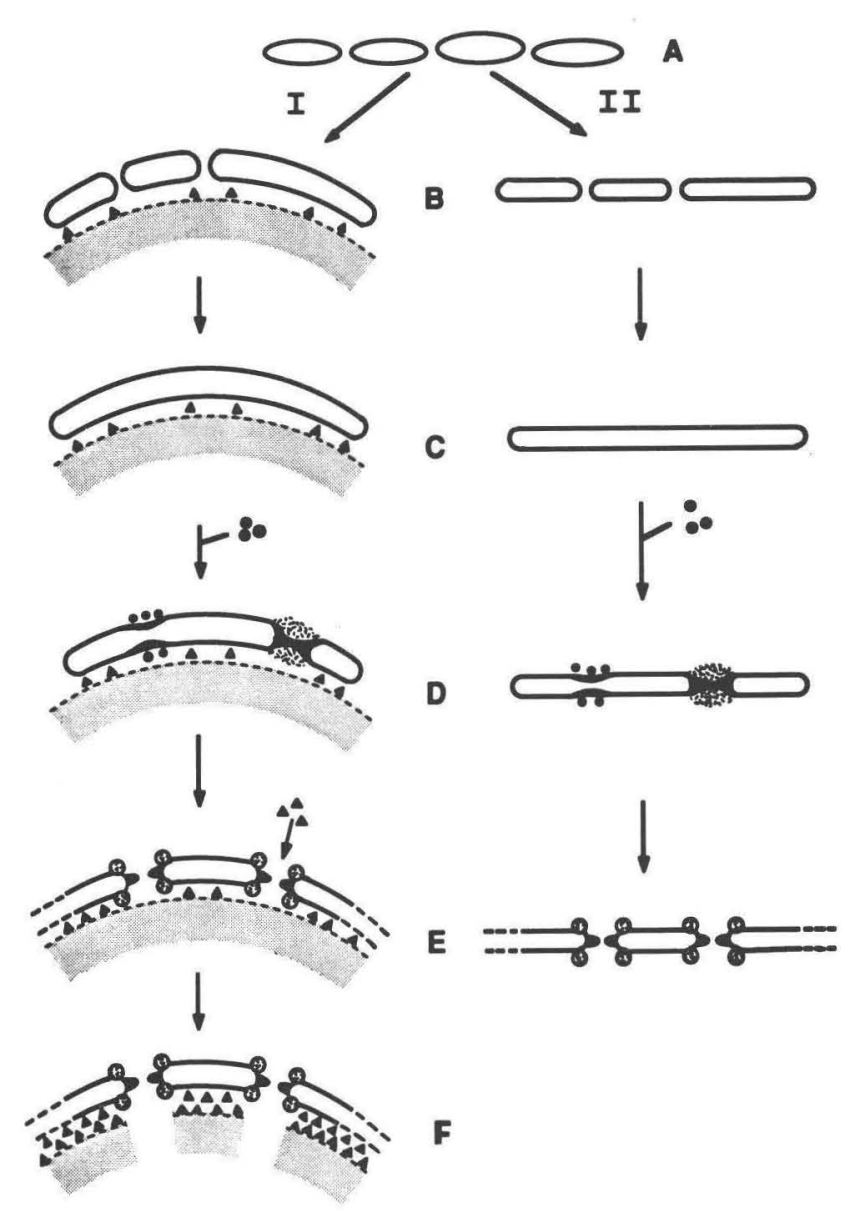

Fig 3. Diagrammatic representation of the assembly of pore complex containing membranes in Xenopus egg extract. Nuclear membrane vesicles which are morphologically distinct from other vesicles by their finely filamentous content [12] are abundant in extract freshly prepared from activated eggs (A). In the presence of chromatin (shaded) and a lamina coating the chromatin surface (triangles), a nuclear envelope will form (pathway I). In the absence of chromatin or in the presence of chromatin lacking a lamina, annulate lamellae will form instead (pathway II). The kinetics of both assembly pathways are identical (for further details see [12]). Fusion of vesicles into flattened membrane cisternae is shown in (B) and (C). The lamina is essential for targeting the vesicles to the chromatin surface; the dispersed arrangement of the triangles indicates that initially only a "minimal lamina" is formed. Soluble pore complex material (filled circles) interact with the nascent membrane cisternae and induce a localized fusionof both membranes ( $D$; from left to right). Finally, the entire chromatin becomes enclosed by a continuous nuclear envelope with numerous pore complexes (E, pathway I). In the absence of chromatin, annulate lamellae are being formed $(\mathrm{E}$, pathway II). The newly formed nuclei then take up soluble lamin proteins through the pore complexes (triangles in E; see also [6]). These imported lamins then become integrated into the already existing "minimal lamina" until the final size of the lamina layer is reached $(F)$. Finally chromatin is reorganized in such a way that chromatin-free channels lead from the pore complexes into the nuclear interior. Preliminary evidence suggests that nuclear envelopes will also form around lamina filaments assembled in vitro in the absence of chromatin (see fig 2 ).

lamina. The role of the lamina is to direct this assembly process to the chromatin surface. Furthermore, our combined data indicate that only a "minimal lamina" is required to target nuclear membrane vesicles to the chromatin surface. Firstly, when nuclei are assembled in p68-depleted extract they become enclosed by a doublemembrane envelope lacking pore complexes and are unable to import protein. However, a lamina is not detectable by immunofluorescence microscopy [11]. Secondly, when lamina assembly is inhibited by addition of antilamin antibodies to the extract, reconstitution of nuclear envelopes no longer occurs at the chromatin surface but rather throughout the cytoplasm in form of annulate lamellae [12]. Hence, a small population of early attaching lamins is sufficient to target membrane vesicles to the chromatin surface and promote nuclear envelope formation. Assembly of a normal nuclear lamina, however, which is clearly visible in reconstituted nuclei by immunofluorescence microscopy $[11,23]$ requires subsequent uptake of soluble lamin proteins through the newly formed pore complexes. By microinjecting synthesized in vitro lamins into Xenopus oocytes, Krohne et al [18] could directly demonstrate that soluble lamins are efficiently transported into the nucleus and integrated into the existing lamina. Furthermore, nuclear import of lamins is also required for postmitotic nuclear remodeling and growth processes accompanying the transition from telophase to G1 in mammalian cells $[3,6]$.

In order to dissect and understand the assembly pathway of the nuclear envelope-pore complex structure, it will be necessary to purify all components involved so that their molecular interactions can be studied separately. Cell-free extracts provide a promising experimental system to reach this goal.

\section{Acknowledgments}

We thank Hilde Merkert for preparation of figure 3 and Georg Krohne (German Cancer Research Center, Heidelberg) for providing antibodies to lamins and purified lamin A. This work received financial support from the Deutsche Forschungsgemeinschaft (SFB 176).

\section{Note added in proof}

The amino acid sequence of the pore-specific protein p68 of Xenopus has been determined by cDNA sequencing and compared with its mammalian homologue p62 (Cordes et al, Eur $J$ Cell Biol 55, 31-47, 1991). A high molecular weight multimeric complex including $\mathrm{p} 62$ has also been identified after detergent extraction of rat liver nuclei (Finlay et al, J Cell Biol 114, 169-183, 1991).

\section{References}

1 Akey CW (1991) The nuclear pore complex: a macromolecular transporter. In: Nucleocytoplasmic Transport (Feldherr $C$, ed) New York: Academic Press (in press)

2 Benavente R, Krohne G (1985) Changes of karyoskeleton during spermatogenesis of Xenopus: expression of lamin $\mathrm{L}_{4}$, a nuclear lamina protein specific of the male germ line. Proc Natl Acad Sci USA 82, 6176-6180

3 Benavente R, Krohne G (1986) Involvement of nuclear lamins in postmitotic reorganization of chromatin as demonstrated by microinjection of lamin antibodies. $J$ Cell Biol 103, 1847-1854

4 Benavente R, Krohne G, Franke WW (1985) Cell typespecific expression of nuclear lamina proteins during development of Xenopus laevis. Cell 41, 177-190 
5 Benavente R, Dabauvalle MC, Scheer U, Chaly N (1989) Functional role of newly formed pore complexes in postmitotic nuclear reorganization. Chromosoma 98, 233-241

6 Benavente R, Scheer U, Chaly N (1989) Nucleocytoplasmic sorting of macromolecules following mitosis: fate of nuclear constituents after inhibition of pore complex function. Eur $J$ Cell Biol 50, 209-210

7 Burke B, Gerace L (1986) A cell free system to study reassembly of the nuclear envelope at the end of mitosis. Cell $44,639-652$

8 Dabauvalle MC, Franke WW (1982) Karyophilic proteins: Polypeptides synthesized in vitro accumulate in the nucleus on microinjection into the cytoplasm of amphibian oocytes. Proc Natl Acad Sci USA 79, 5302-5306

9 Dabauvalle MC, Franke WW (1986) Determination of the intracellular state of soluble macromolecules by gel filtration in vivo in the cytoplasm of amphibian oocytes. $J$ Cell Biol 102, 2006-2014

10 Dabauvalle MC, Benavente R, Chaly N (1988) Monoclonal antibodies to a $M_{\mathrm{r}} 68000$ pore complex glycoprotein interfere with nuclear protein uptake in Xenopus oocytes. Chromosoma 97, 193-197

11 Dabauvalle MC, Loos K, Scheer U (1990) Identification of a soluble precursor complex essential for nuclear pore assembly in vitro. Chromosoma 100, 56-66

12 Dabauvalle MC, Loos K, Merkert H, Scheer U (1991) Spontaneous assembly of pore complex-containing membranes ("'annulate lamellae") in Xenopus egg extract in the absence of chromatin. J Cell Biol 112, 1073-1082

13 Feldherr CM, Akin D (1990) EM visualization of nucleocytoplasmic transport processes. Electron Microsc Rev 3, 73-86

14 Finlay DR, Forbes DJ (1990) Reconstitution of biochemically altered nuclear pores: transport can be eliminated and restored. Cell $60,17-29$

15 Gerace L, Burke B (1988) Functional organization of the nuclear envelope. Annu Rev Cell Biol 4, 335-374

16 Glass JR, Gerace L (1990) Lamins A and C bind and assemble at the surface of mitotic chromosomes. J Cell Biol $111,1047-1057$

17 Greber UF, Senior A, Gerace L (1990) A major glycoprotein of the nuclear pore complex is a membrane-spanning polypeptide with a large lumenal domain and a small cytoplasmic tail. EMBO J 9, 1493-1502

18 Krohne G, Waizenegger I, Höger TH (1989) The conserved carboxy-terminal cysteine of nuclear lamins is essential for lamin association with the nuclear envelope. J Cell Biol 109, 2003-2011

19 Lohka MJ (1988) The reconstitution of nuclear envelopes in cell-free extracts. Cell Biol Int Rep 12, 833-848
20 Lohka MJ, Masui Y (1983) Formation in vitro of sperm pronuclei and mitotic chromosomes induced by amphibian ooplasmic components. Science 220, 719-721

21 Lohka MJ, Masui Y (1984) Roles of cytosol and cytoplasmic particles in nuclear envelope assembly and sperm pronuclear formation in cell-free preparations from amphibian eggs. J Cell Biol 98, 1222-1230

22 Newmeyer DD, Lucocq JM, Bürglin TR, De Robertis EM (1986) Assembly in vitro of nuclei active in nuclear protein transport: ATP is required for nucleoplasmin accumulation. EMBO J 5, 501-510

23 Newport J (1987) Nuclear reconstitution in vitro: stages of assembly around protein-free DNA. Cell 48, 205-217

24 Newport J, Wilson KL, Dunphy WG (1990) A laminindependent pathway for nuclear envelope assembly. J Cell Biol 111, 2247-2259

25 Reichelt R, Holzenburg A, Buhle EL, Jarnik M, Engel A, Aebi U (1990) Correlation between structure and mass distribution of the nuclear pore complex and of distinct pore complex components. J Cell Biol 110, 883894

26 Scheer U, Dabauvalle MC, Merkert H, Benavente R (1988) The nuclear envelope and the organization of the pore complexes. Cell Biol Int Rep 12, 669-689

27 Senior A, Gerace L (1988) Integral membrane proteins specific to the inner nuclear membrane and associated with the nuclear lamina. J Cell Biol 107, 2029-2036

28 Sheehan MA, Mills AD, Sleeman AM, Laskey RA, Blow JJ (1988) Steps in assembly of replication-competent nuclei in a cell-free system from Xenopus eggs. J Cell Biol 106, $1-12$

29 Starr CM, Hanover JA (1990) Structure and function of the nuclear pore complex: new perspectives. BioEssays 12, $323-330$

30 Starr CM, D’Onofrio M, Park MK, Hanover JA (1990) Primary sequence and heterologous expression of nuclear pore glycoprotein p62. J Cell Biol 110, 1861-1871

31 Stick R, Hausen P (1985) Changes in the nuclear lamina composition during early development of Xenopus laevis. Cell $41,191-200$

32 Wilson KL, Newport J (1988) A trypsin-sensitive receptor on membrane vesicles is required for nuclear envelope formation in vitro. $J$ Cell Biol 107, 57-68

33 Wolin SL, Krohne G, Kirschner MW (1987) A new lamin in Xenopus somatic tissues displays strong homology to a human lamin A. EMBO J 6, 3809-3818

34 Wozniak RW, Bartnik E, Blobel G (1989) Primary structure analysis of an integral membrane glycoprotein of the nuclear pore. J Cell Biol 108, 2083-2092 